\title{
¿EMPLEAMOS EFICIENTEMENTE LAS TECNOLOGÍAS DE LA INFORMACIÓN EN LA ADMINISTRACIÓN PÚBLICA?
} Are we using information technologies in the public administration efficiently?

\author{
Victor Miranda Alfaro ${ }^{1}$ \\ Universidad Alas Peruanas, Perú
}

\section{RESUMEN}

Toda Administración requiere de herramientas que sean el valor agregado a la capacidad de las personas, es ahí donde las Tecnologías de la Información constituyen la herramienta necesaria e imprescindible para una organización eficiente, acorde a la transformación de la tecnología. Así mismo el diseño, adaptación y su posterior operación requieren de una gran inversión y consumo, sin embargo esto no satisface a las expectativas de los directivos ya que el retorno de la inversión, no siempre es evidente, normalmente a falta de una estadística que demuestre la verdadera importancia de las Tecnologías de la Información. El Estado cumple un papel importante en todo proceso de transformación de un país. El Estado es la mayor organización que provee y recolecta la información, los servicios que proporcionan deben ser accesibles a la población, especialmente, a los sectores más desfavorecidos. La sociedad exige más y mejores servicios, en la acelerada transformación de la tecnología y los avances en las Tecnologías de la Información y las Comunicaciones (TIC), plantean una serie de desafíos en la sociedad y la estructura gubernamental de los países.

Palabras Clave: TIC, administración pública, sociedad de la información, gestión pública.

\section{Abstract}

Any Administration needs of tools that they are the value added to the capacity of the persons, is there where the Technologies of the Information constitute the necessary and indispensable tool for an efficient, identical organization to the transformation of the technology. Likewise the design, adjustment and his later operation need of a great investment and consumption, nevertheless this does not satisfy to the expectations of the executives since the return of the investment, not always it is evident, normally for lack of a statistics that demonstrates the real importance of the Technologies of the Information. The State fulfills an important paper in any process of transformation of a country. The State is the major organization that provides and gathers the information, the services that they provide must be accessible to the population, specially, to the most disadvantaged sectors. The society demands more and better services, in the intensive transformation of the technology and the advances in the Technologies of the Information and the Communications (TIC), they raise a series of challenges in the society and the governmental structure of the countries.

Keywords: TIC, public administration, society of the information, public management. 


\section{INTRODUCCIÓN}

Las Tecnologías de la Información y Comunicación se han desarrollado vertiginosamente desde su inicio, lo que en un primer momento se tomó como una herramienta para optimizar los procesos administrativos, esto tomo preponderancia ya que hoy es un instrumento importante y estratégico para los diversos campos de la actividad humana.

La transformación tecnológica en el mundo de las Tecnologías de la Información y las Comunicaciones (TIC), han cambiado y continúan cambiando la sociedad. Este proceso tecnológico presenta diferentes y múltiples alternativas, como consecuencia de los distintos matices que ofrece. Sin embargo, este progreso hace que nos enfrentemos a las consecuencias de un empleo inadecuado, intencionado o no, de estas tecnologías, en todos los niveles, estatal, empresarial, académico, poblacional entre otros.

Dirigir y aplicar las TIC a los procesos de negocio requiere, necesariamente, disponer de personas responsables y capacitadas para asumir responsabilidades. Si las personas cometen errores, entonces no existirá una tecnología y método capaz de incrementar el valor a los procesos de negocio. Toda organización evoluciona cuando lo dirige un grupo humano; incluso en las corporaciones más tradicionales, la resistencia al cambio constituye un serio riesgo de falta de eficiencia, eficacia y credibilidad. Toda mejora y transformación se interrelacionan cuando los individuos encuentran nuevos empleos para la tecnología

Como es de suponer en las dependencias públicas y entidades privadas, las áreas de gestión financiera no perciben el efecto multiplicador del empleo de las Tecnologías de la Información y las Comunicaciones (TIC) o cómo estas podrían mejorar la eficacia y eficiencia de sus actividades de negocio. Es decir, se desconoce el valor de las TI, pues no existen parámetros de medición relativos al desempeño, antes, durante y después de su aplicación, teniendo en consideración lo siguiente:

a. La mano de obra que se ahorra.

b. El tiempo que reducen los plazos, en la ejecución de un proceso o actividad realizada. c. Aspectos innovadores en la información que se han desarrollado para un negocio.

d. Capacidad disponible para un nuevo proceso.

Por ejemplo, un servicio de enseñanza a distancia en comparación con un sistema de enseñanza presencial proporciona algunas facilidades y/o beneficios siguientes:

a. Ahorro de costos en movilidad y alojamiento del personal

b. Reducción de costos en lo relacionado a la infraestructura física (aulas, servicios básicos, climatización, entre otros)

c. Reducción de los costos del material educativo (mobiliario, informática, textos, entre otros)

d. Reducir la cantidad del personal especializado en tareas académicas, así como en actividades administrativas.

e. Capacidad de alinear en cada momento el número de empleados formados con las necesidades actuales de las unidades de negocio, eludiendo el riesgo de sobrecostos derivados de capacitar a más personal del necesario, o viceversa.

f. Formación académica constante y actualizada del personal a costos reducidos.

Los anteriores son indicadores muy importantes en las Tecnologías de la Información, estos beneficios son de carácter inmediato para su ejecución y para la adecuada gestión económica; por consiguiente, serán estas unidades de negocio los únicos departamentos corporativos en disposición de conocer y medir la utilidad y las garantías proporcionadas por el empleo de las TI y serán ellas mismas quienes pueden liderar la evolución de los servicios y sistemas, ya que, normalmente, el departamento y el personal especialista de TI desconocen esta esos datos, indicadores y mediciones capaces de calibrar el impacto y la rentabilidad de los servicios y sistemas.

El nivel de dependencia tecnológica en toda organización moderna, ya sea pública o privada, no ha dejado de crecer. En los procesos o líneas de negocio, los trabajadores de la empresa están en contacto con dispositivos y componentes del mundo de las Tecnologías de la Información desde el inicio de labores diarias. Por consiguiente, 
las compañías, y sus procesos de negocio, experimentan una alta dependencia de la tecnología, asociada con sus recursos e infraestructura. Esta transformación marca la transición entre la era industrial y la era de la información, donde todo está interrelacionado y funciona de una manera flexible y dinámica.

\section{MÉTODO}

Para la revisión de la literatura se ha consultado la bibliografía necesaria relacionada al tema, de investigadores dedicados a aportar la aplicación de las tecnologías en la administración pública, encontrándose aspectos que facilitan la elaboración del artículo.

\section{TRADICIÓN E INNOVACIÓN: AL ENCUENTRO}

Cualquier organización con años de historia es heredera de costumbres, tradiciones y modelos de gestión que se han ido formando a lo largo de los ańos como consecuencia de su visión, misión, objetivos y de la coyuntura específica a las que se ha debido responder en cada momento de su historia. Por supuesto, el capital humano, con sus cualidades, actitudes, y limitaciones, con su maravillosa imaginación y creatividad, constituyendo elementos cruciales en la cultura de cada organización.

La irrupción de las TIC en los entornos corporativos presenta la característica diferencial de constituir un elemento exógeno que, excepto en el caso de las organizaciones cuyo "leitmotiv" y negocio es la propia tecnología, puede amenazar o modificar los modelos ordinarios de funcionamiento de las corporaciones. Así pues, a la hora de administrar estas nuevas herramientas y capacidades, parece una comprensible respuesta humana que cada organización procure adoptar modelos de gobierno y de gestión de sus TI basados en la extrapolación de los mismos modelos con los que históricamente viene administrando sus procesos de negocio tradicionales.

La Administración Pública normalmente necesita innovar sus prácticas y su forma de organizar sus capacidades relacionadas con las TIC, así como evolucionar su normatividad; el protagonismo no corresponde a los recursos basados en tecnología, sino a la capacidad de las TIC para hacer crecer los procesos de negocio. "La tecnología facilita y soporta el Negocio" debe ser sustituida hoy día por "La tecnología es el Negocio"2.

La administración pública tiene un papel muy importante en el desarrollo de la Sociedad de la Información en dos áreas principales:

a. Como usuaria de las Tecnologías de la Información y Comunicación, con el fin de mejorar la calidad de los servicios públicos, profundizando la transparencia de su intervención, promoviendo la participación ciudadana.

b. Como dinamizador de la Sociedad de la Información, formulando y ejecutando políticas que promuevan la inclusión de las TIC en la sociedad y su ingreso en todos los grupos sociales.

Mientras las organizaciones son de pequeña dimensión o se mantienen dentro de un limitado nivel de madurez en cuanto al uso de las TI, generalmente orientado normalmente a la administración de plataformas tecnológicas, el problema puede tener un impacto moderado, cuando las características del entorno son otras y las TI se convierten en innovaciones del siglo XXI, las respuestas y modelos de gestión cotidianos de la organización dejan de ser válidos si se quiere convertir en valor cualquier inversión en estos activos. En ese escenario las TI precisan de unos modelos especializados de gobierno y gestión basados en los conocimientos y experiencias de los profesionales de todo el mundo que moldean los marcos de referencia normalizados. La adaptación de las TI a la idiosincrasia de cada organización no se encauzará a través de la utilización de los mismos modelos de gestión que se pudieran utilizar. El proceso de inmersión de las TI en la cultura y en la idiosincrasia de cada organización se logra definitivamente cuando las TI son un elemento más en el diseño y funcionamiento de cada uno de sus procesos de negocio: un actor indispensable y consustancial a los mismos que, definitivamente, ya han superado la condición de elemento externo de apoyo. De ese modo, tradición e innovación se dan la mano.

2 Rafael Achaerandio, analista de la consultora IDC España, en el evento CIO Directions 2013 
En algunas organizaciones públicas o privadas, las unidades de negocio no perciben el efecto multiplicador de las TIC o como éstas pueden mejorar la eficacia y eficiencia de sus actividades de negocio. Es decir, no se conoce el valor de las Tecnologías de la Información, ya que, generalmente, no existen mediciones relativas al desempeño antes y después de su aplicación, como por ejemplo: Cantidad de mano de obra que se ahorra, plazos de tiempo que se reducen en un proceso o actividad, empleo de métodos novedosos de la información que se desarrollan para el negocio, Capacidad innovadora disponible.

\section{LAS TIC Y CÓMO ABORDAR LAS DIFERENCIAS DE INTERESES ENTRE LA PROPIEDAD Y LA ADMINISTRACIÓN DE UNA EMPRESA}

El concepto de "gobierno corporativo de TI", o "gobernanza de las TI", encuentra su origen en el de "gobernanza", entendido éste como el marco para la buena gestión de los recursos por parte de los gobiernos y para la promoción de la participación de la sociedad. En las TI se emplea el término "Gobernanza", que en la actualidad este concepto, tiene su connotación más académica sobre la transformación que sufre el Estado, sin embargo, los términos "Gobernabilidad y Gobernanza" se asocian a la "Administración Pública", mediante el cual la gestión se realiza bajo principios de honestidad, transparencia, eficiencia, eficacia en los recursos públicos.

Asociado al término "gobernanza", emerge el de "gobierno corporativo" como mecanismo para abordar las diferencias de intereses entre la propiedad y la administración de una empresa; en particular, el "Gobierno Corporativo" hace referencia a mecanismos que previenen el posible conflicto de interés y de control y salvaguarda por parte de los accionistas sobre las acciones realizadas por los miembros del consejo de administración.

La definición más famosa de gobierno corporativo es la que se formuló en 1992 por Sir Adrián Cadbury en el informe sobre los aspectos financieros del Gobierno Corporativo en el Reino Unido, Sin embargo es importante destacar "El término Gobierno Corporativo, acepta definiciones tanto amplias como estrechas. (...) Lo importante es destacar que el gobierno corporativo no es un instrumento individual sino más bien un concepto que incluye el debate sobre las estructuras apropiadas de gestión y control de las empresas. También incluye las reglas que regulan las relaciones de poder entre los propietarios, el consejo de administración, la administración y las partes interesadas tales como los empleados, proveedores, clientes y público en general ${ }^{3}$.

En el año 2004, los investigadores del Instituto de Tecnología de Massachusetts (MIT), Peter Weill y Jeanne Ross propondrían seis activos clave que deben ser objeto de prácticas de gobierno en cualquier organización ${ }^{4}$ :

a. Los Activos financieros.

b. Los Activos físicos.

c. Los Recursos humanos.

d. El Esquema de relaciones de una organización.

e. La Propiedad intelectual.

f. Las TIC5.

En el universo específico de las TI, todos estos avances se verían consolidados especialmente en dos marcos de referencia: El trabajo integral de los Objetivos de Control para Información y Tecnologías Relacionadas $(\mathrm{COBIT})^{6}$ para el gobierno y la gestión de las TI de la empresa, actualmente en su versión 5, y la norma internacional UNE-ISO/IEC 38500 "Gobernanza corporativa de la Tecnología de la Información”7. Como no podría ser de otra forma en el actual estado de madurez y conocimiento de las organizaciones modernas, ambos establecen una clara distinción entre gobierno y gestión. Según reza uno de los cinco principios claves de COBIT 5, "Separar el Gobierno de la Gestión", "estas dos disciplinas engloban diferentes tipos de actividades, que requieren diferentes estructuras organizativas y sirven a diferentes propósito"s":

\footnotetext{
3 NR Narayana Murthy, presidente del Comité sobre Gobierno Corporativo, Junta de Valores y Bolsas de India, 2003

4 Peter Weill and Jeanne Ross., IT Governance: How Top Performers Manage IT Decision Rights for Superior Results. Harvard Business Press. 2003

5 http://www.magazcitum.com.mx

6 Control Objectives for Information and related Technology

7 International Organization for Standardization, ISO, Comisión Electrotécnica Internacional, IEC

8 www.isaca.org/cobit
} 
- El Gobierno: permite que se evalúan las múltiples necesidades, condiciones y opciones en las partes interesadas para alcanzar las metas corporativas que estas sean equilibradas; identificando el norte a seguir a través de priorizar y la buena toma de decisiones.

- La Gestión: diseña, planifica, construye, ejecuta y controla las actividades trazadas en la dirección establecida por el directorio gubernamental, con la finalidad de alcanzar las metas definidas para la organización.

Los principios que expresan el comportamiento deseable para orientar la toma de decisiones, indica lo que debería suceder, pero no prescribe cómo, cuándo o por quien se pondría en práctica, ya que esos aspectos dependerán de la naturaleza de la organización que los implanta. Los seis principios sobre los que se aplicarán las tres tareas del modelo (Evaluar, Dirigir y Monitorizar) son:

a. Responsabilidad: Individuos y grupos deben comprender y aceptar las responsabilidades con respecto a la demanda y al suministro de productos y servicios de TI.

b. Estrategia: La estrategia en una organización considera sus fortalezas actuales y futuras de las TI, su planeación debe satisfacer las necesidades actuales y futuras en la estrategia del negocio.

c. Adquisición: Toda adquisición de las TI se deben realizar por razones técnicas, sobre la base de un análisis adecuado, toma de decisiones claras y transparentes.

d. Desempeño: Las TI deben satisfacer el propósito de dar soporte a la organización, mediante la provisión de servicios, niveles de servicio y calidad de servicio requeridos para alcanzar los requisitos presentes y futuros del negocio.

e. Cumplimiento: Las TI cumplen con toda la legislación y normativas obligatorias.

f. Conducta humana: Las políticas de TI, prácticas y decisiones relacionadas con las TI deben mostrar respeto hacia la conducta humana, incluyendo las necesidades actuales y futuras de todas las personas implicadas en el proceso.

Finalmente, el modelo se articula a través de tres tareas principales: a. Evaluar, su empleo en la actualidad y prever el futuro de las TI.

b. Dirigir, el planeamiento, y ejecución de los planes a desarrollar las políticas para asegurar el uso adecuado y eficiente de las TI, las cuales satisfaga los objetivos de la organización.

c. Monitorizar, que las políticas sean cumplidas de acuerdo a lo planificado.

\section{CONCLUSIONES}

La introducción de las TIC en la administración pública, generan impactos en la estructura y los procesos administrativos, este producto es relevante en el ámbito de los recursos humanos y la cultura organizacional. Por ello es importante tomar en consideración las necesidades de formación y las repercusiones que la introducción del cambio genera en las expectativas del funcionario público dentro de la organización.

La accesibilidad electrónica a los servicios públicos y su ejecución en la Administración Pública, utilizando las Tecnologías de la Información, facilitará que se logre una eficiencia y efectividad en el aparato del Estado logrando que las gestiones que realizan los usuarios sean rápidas y con seguridad, permitiendo en el empleado público mayor productividad y al estado menores costos en insumos y personal, dejando en lo posible de prescindir del excesivo papeleo y muchas ventanillas de atención (ventanilla única de atención).

El uso universal de las Tecnologías de la Información Comunicaciones, no sólo simplifican la vida de los ciudadanos; son también una herramienta que mejora las condiciones de vida de la población cuyo fin último es el de reducir la pobreza. Quizás sea un objetivo ambicioso para un ámbito de la realidad social como es el de la tecnología, sin embargo, no podemos negar que las ventajas y los beneficios que implican la mejora de la gestión pública y la transparencia sí pueden mejorar en última instancia la distribución de la riqueza así como la mejora en la recaudación de impuestos Locales, Regionales y Nacionales. 


\section{REFERENCIAS BIBLIOGRÁFICAS}

Abelló, H (2003). Ciudadanía y gobierno electrónico: ¿̨hacia una nueva democracia? Gobierno digital: tendencias y desafíos. Bogotá: Universidad Externado de Colombia, 131146.

Arroyo, A. (2013). Origen y evolución del concepto de "Gobierno Corporativo de TI". Recuperado de http://www.magazcitum.com.mx/

Castells, M. (2006). La Era de la Información. Ed. Siglo XXI, Tercera edición, Madrid, Volúmen III.

CA Technologies. Informe The Future Role of the CIO; Digital Literacy, Recuperado de http://bit.ly/NXX1lg

Cohen, W. M. \& Levinthal, D.A., (1989). 'Innovation and Learning: The Two Faces of R\&D' [Innovación y aprendizaje: las dos Caras de I+D] The Economic Journal, England, pp $569-596$.

González, E. (2012). Análisis Ético del Informe Cadbury, Revista de la Universitat Jaume I (Castellón), Pag 5-6.

Lanza, Mario (2003). Algunas reflexiones y consideraciones sobre gobierno electrónico. Modernización de las finanzas públicas. Revista Centroamericana de Administración Pública. Instituto Centroamericano de Administración Pública ICAP., enero-diciembre. P. 119-129

Martínez, A. \& Corrales, M. (Coords.). (2010). Administración de conocimiento y desarrollo basado en conocimiento: redes e innovación. México, D.F.: Cengage Learning

Molina, Marlon. Blog de Marlon Molina, el ser humano que usa la tecnología. La Tecnología de la Información no es un vertical en la empresa. Recuperado de http://marlonmolina.tecnofor.es/

Rockett, Lori. (2003) "Las TI importan, si uno quiere". Revista de la Business School Harvard Business Review, USA, http://www.iese.edu/es/files/Art_EN_Rocket_ITMatters_ Oct03_ESP_tcm5-7433.

Weill, P. y Ross, J. (2004) IT Governance: How Top Performers manage IT Decision Rights for Superior Results, Editorial Harvard Business Press, USA, pp 50-269. 